\title{
A New Fractional Order Hold and Its Capability in Frequency Response and Zero Placement
}

\author{
Farzad Hashemzadeh, ${ }^{1}$ Amin Rezaei Pish-Robat, ${ }^{2}$ and Parviz Jabehdar-Maralani ${ }^{3}$ \\ ${ }^{1}$ Faculty of Electrical and Computer Engineering, University of Tabriz, Tabriz, Iran \\ ${ }^{2}$ Faculty of Electrical and Computer Engineering, Bonab University, Bonab, Iran \\ ${ }^{3}$ School of Electrical and Computer Engineering, University of Tehran, Tehran, Iran
}

Correspondence should be addressed to Farzad Hashemzadeh; farzad.hashemzadeh@ualberta.ca

Received 17 March 2013; Revised 12 June 2013; Accepted 2 July 2013

Academic Editor: Hak-Keung Lam

Copyright (C) 2013 Farzad Hashemzadeh et al. This is an open access article distributed under the Creative Commons Attribution License, which permits unrestricted use, distribution, and reproduction in any medium, provided the original work is properly cited.

This paper introduces a new holder with application on digital control systems. This holder is a combination of fractional order hold $(\mathrm{FROH})$ and zero-order hold $(\mathrm{ZOH})$ that has the capability of both holders and a frequency response better than both of $\mathrm{ZOH}$ and FROH. For the stability of zeros of the sampled system two theorems are stated and proved with the assumption that the sampling period is very small. Also simulation results are studied to show the effectiveness of the proposed holder and better performance results in comparison with $\mathrm{ZOH}$ and $\mathrm{FROH}$.

\section{Introduction}

In digital control systems, the output of the system is sampled by an analog-to-digital converter (ADC) and then compared with digital reference inputs that are generated by the computer. The difference is given to the controller, and the output of the controller needs to be given to a holder block in order to give to the continuous time system. The holder block generates continuous time signal between sampling times, and the more common and usual holders are $\mathrm{ZOH}$ and $\mathrm{FOH}$. The $\mathrm{ZOH}$ is the most common holder used as digital-toanalog converter (DAC) in industry. FROH is similar to first order hold (FOH) but has a variable slope [1], and due to an adjustable parameter, it has a suitable capability for placement of additional produced zeros [2]. Holders such as $\mathrm{ZOH}$ and FOH which are used in digital control systems sometimes generate additional non-minimum-phase zeros in $z$-domain [2]. In the design of a feedback control system, zeros play an important role, and most of the design methods based on pole-zero cancellation require that zeros of the system be stable. However, in discretizing continuous time systems with sample and hold, the stability of zeros of the sampled system is not necessarily preserved. Thus it is necessary to know the conditions under which we can obtain a minimum-phase sampled system. Depending on the relative degree of the system, $\mathrm{FOH}$ generates non-minimum-phase zeros and $\mathrm{ZOH}$ maps additional generated zeros to unit circle in $z$-plane. The number of the additional generated zeros depends on the relative degree of the system. Both $\mathrm{FOH}$ and $\mathrm{ZOH}$ cannot control the location of additional generated zeros. Based on disadvantages of $\mathrm{ZOH}$ and $\mathrm{FOH}, \mathrm{FROH}$ was introduced to have non-minimum-phase system. Using adjustable slope of holder in $\mathrm{FROH}$, very small sampling time guarantees that additional generated zeros of the sampled systems are minimum-phase in the system with relative degrees of 0,1 , and 2 .

Replacing $\mathrm{ZOH}$ with FROH in a digital control system, the ability to improve the stability of zeros of system is shown in [2]. Compared with $\mathrm{ZOH}$, the stability of zeros of the sampled system can be improved by using FROH, and the conditions under which the $\mathrm{FROH}$ has better performance than $\mathrm{ZOH}$ have been clarified theoretically in [3]. Most of the researches on the properties of zeros of the sampled system are made under the assumption that $\mathrm{ZOH}, \mathrm{FOH}$, or the FROH is employed [4-6].

In order to have minimum-phase additional generated zeros, when using FROH in the systems with relative degree one, the slope of the holder $(\beta)$ should be $\beta>-1$ and in 
systems with relative degree two, the slope of the holder should be $-1<\beta<0$ [5].

In this paper, a new holder to be applied to digital control systems is introduced; we named it mixed fractional order hold (MFROH). MFROH is a combination of FROH and $\mathrm{ZOH}$ that has the capabilities of both holders and has a frequency response better than $\mathrm{ZOH}$ and $\mathrm{FROH}$. MFROH places additional generated zeros better than $\mathrm{FROH}$ and $\mathrm{ZOH}$. The number of these additional zeros depends on the relative degree of the system, and hence it is difficult to design a digital controller for systems with large relative degrees [3]. MFROH has two adjustable parameters and has the same capability as FROH with respect to placement of additional generated zeros for different relative degrees and increases the range of acceptable slope of the holder, therefore it improves the capability of the holder in zero placements. At low frequencies, $\mathrm{FROH}$ is a better approximation to an ideal low pass filter than $\mathrm{ZOH}$, but at high frequencies, $\mathrm{ZOH}$ is better than $\mathrm{FROH}$. Because of the fact that $\mathrm{MFROH}$ is a combination of $\mathrm{ZOH}$ and $\mathrm{FROH}$, it has the same behavior as FROH at low frequencies and the same behavior as $\mathrm{ZOH}$ at high frequencies, and hence by tuning its two parameters, we can have a better frequency response than $\mathrm{ZOH}$ and $\mathrm{FROH}$. Therefore, with adjusting the parameters of $\mathrm{MFROH}$, the amplitude of frequency response of $\mathrm{MFROH}$ would be very similar to ideal low-pass filter. MFROH has the capability that it can be converted to $\mathrm{ZOH}$ or FROH by tuning one of its parameters.

In Section 2 the structure of MFROH is introduced, and the frequency response of $\mathrm{MFROH}$ is given in Section 3. Stability studies of zeros in sampled system and two theorems for stability of zeros are stated and proved in Section 4. In Section 5 simulation results are studied, and conclusion is stated in Section 6.

\section{The Structure of MFROH}

MFROH is a line with slope $\beta$ over the interval $[k T, k T+\Delta T]$ that coincides with the output at instant $t=k T$. Also it is a line with zero slope over the interval $[k T+\Delta T, k T+T]$ with the continuity preserving at $t=k T+\Delta T$. A schematic of MFROH is shown in Figure 1 and is described by the following equations:

$$
u(t)=\left\{\begin{array}{c}
u(k T)+\beta \frac{u(k T)-u((k-1) T)}{T}(t-k T) \\
k T \leq t \leq(k+\Delta) T \\
u(k T)+\beta \frac{u(k T)-u((k-1) T)}{T}(\Delta T) \\
(k+\Delta) T \leq t \leq(k+1) T \quad 0 \leq \Delta \leq 1,
\end{array}\right.
$$

where $T$ is the sampling time, $\beta$ is the adjustable parameter of holder slope over the interval $[k T, k T+\Delta T]$, and $\Delta$ is the adjustable parameter describing the location of slope variation over interval $[k T, k T+\Delta T]$. The impulse response of $\mathrm{MFROH}$ according to (1) is shown in Figure 2. According to

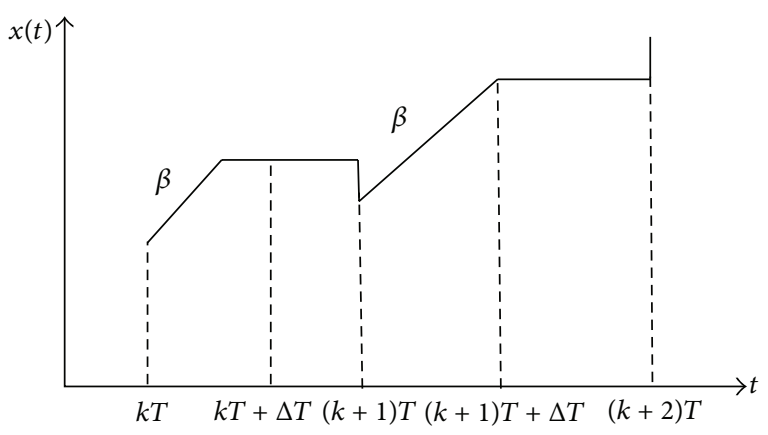

FIgURE 1: Samples in MFROH.

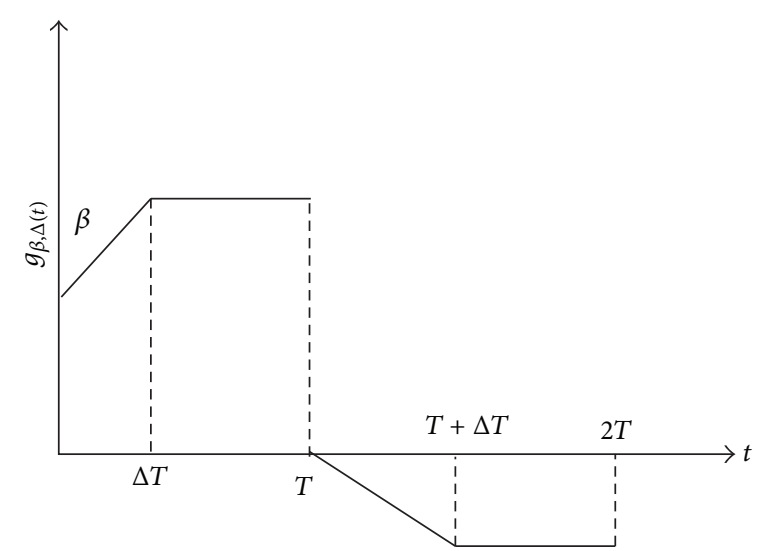

FIGURE 2: Impulse response of MFROH.

(1) and Figure 2, the transfer function of $\mathrm{MFROH}$ is given by the following equation:

$$
\begin{aligned}
G_{\beta, \Delta} & =\frac{\left(1-e^{-s T}\right)\left(1-\Delta \beta e^{-s T}\right)}{s}+\frac{\beta\left(1-e^{-s T}\right)\left(1-e^{-\Delta s T}\right)}{T s^{2}} \\
& =\frac{\left(1-e^{-s T}\right)}{s}\left\{\left(1-\Delta \beta e^{-s T}\right)+\frac{\beta\left(1-e^{-\Delta s T}\right)}{T s}\right\} .
\end{aligned}
$$

In [7], implementation of FROH is discussed using a lot of $\mathrm{ZOH}$. Given MFROH is the combination of $\mathrm{ZOH}$ and $\mathrm{FROH}$, according to [7] MFROH can be implemented using $\mathrm{ZOH}$ blocks in practical application. The new proposed holder device has limits in frequency synthesis methods based on delta, bilinear, or tustins transformations.

\section{Frequency Response of MFROH}

In a discrete time system the high frequency terms of sampled signal that are generated in the sampling process must be attenuated before applying the signal to continuous time systems. In order to attenuate the high frequency terms of a sampled signal, it is usually fed to a hold circuit. Hold circuit is a low-pass filter that converts the sampled signal to a continuous-time signal. An ideal low-pass filter or an ideal holder converts sampled signal to continuous-time signal with no 


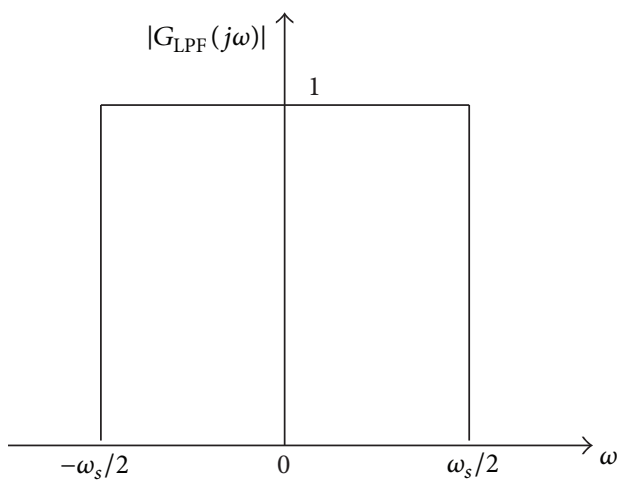

FIGURE 3: Frequency response of an ideal filter or an ideal holder.

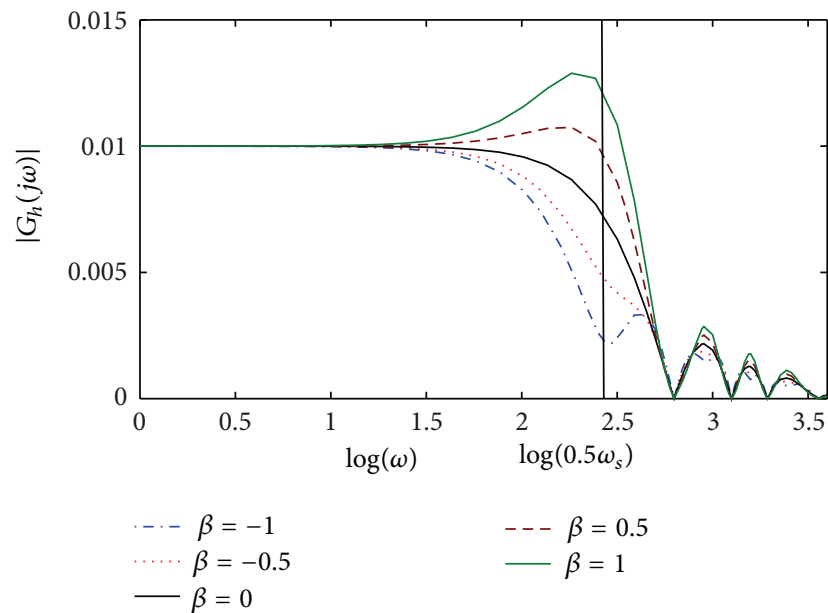

FIGURE 4: Amplitude of frequency responses of $\mathrm{ZOH}$ and $\mathrm{FROH}$ for different values of $\beta$.

high frequency terms, but in the real world there is no such an ideal filter [8].

3.1. Frequency Response Specifications. Sampling process generates many additional terms in addition to the main term, and the ideal filter attenuates all these additional terms to zero and only passes the main term. Therefore, a practical filter is such a filter that passes the main term without any frequency distortion and attenuates all additional terms as much as possible. This means that the frequency response of practical filter must resemble to that of the ideal filter in Figure 3 as much as possible.

The amplitudes of frequency responses of $\mathrm{ZOH}$ and $\mathrm{FROH}$ for different values of $\beta$ with sampling time $T=$ $0.01 \mathrm{sec}$ are shown in Figure 4.

Regarding Figure 4 , for negative values of $\beta$ and reducing $\beta$, the amplitude of frequency response at $\omega=\omega_{s} / 2$ is reduced dramatically, and it is not smooth and has large peaks after its first minimum, that is, for $\beta=-1$. The amplitude of frequency response increases after its first minimum in $\omega=$ $\omega_{s}$, and the filter passes undesirable signals generating from sampling around $\omega=\omega_{s}$ and leads to frequency aliasing. Therefore, the optimal value of $\beta$ should be chosen among the
TABLE 1: FROH's amplitude of the frequency responses for different values of $\beta$ at $\omega=\omega_{s} / 2$.

\begin{tabular}{lccccc}
\hline$\beta$ & -1 & -0.4 & 0 & 0.4 & 1 \\
\hline$\left|G_{h}\left(\left(j \omega_{s}\right) / 2\right)\right| \times 10^{3}$ & 3.96 & 4.17 & 6.4 & 9.13 & 13.4 \\
\hline
\end{tabular}

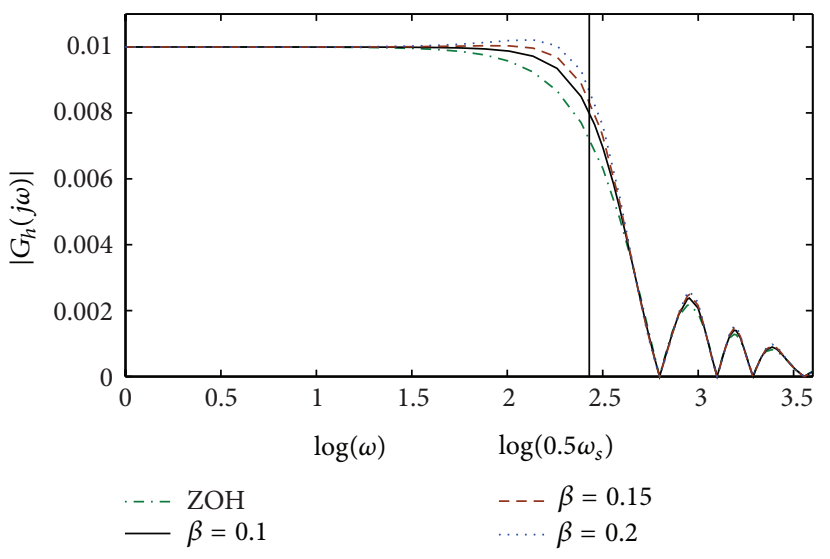

FIGURE 5: Amplitude of the frequency responses of $\mathrm{ZOH}$ and $\mathrm{FROH}$ for small $\beta$.

positive values of $\beta$ in FROH. By comparing the amplitudes of frequency responses of $\mathrm{ZOH}$ and $\mathrm{FROH}$, it can be seen from Table 1 that for positive values of $\beta$ at $\omega=\omega_{s} / 2$, the decrease in the $\mathrm{ZOH}$ is much more than the decrease in the FROH.

In Figure 5, the amplitudes of the frequency responses of $\mathrm{ZOH}$ and $\mathrm{FROH}$ for small values of $\beta$ are shown. In low frequencies, the transfer function of $\mathrm{FROH}$ has a better approximation of ideal low-pass filter than the transfer function of $\mathrm{ZOH}$, because at low frequencies the amplitude of the frequency response of $\mathrm{ZOH}$ is reduced much more than $\mathrm{FROH}$, but at high frequencies $\mathrm{ZOH}$ is better.

The amplitudes of the frequency response of $\mathrm{MFROH}$ for different values of $\beta$ and $\Delta$ are shown in Figure 6. According to Figure 6 and based on two adjustable parameters of $\mathrm{MFROH}$, it is possible to adjust $\beta$ and $\Delta$ so that the amplitude of the frequency response of MFROH becomes very similar to that of the ideal low-pass filter that is shown in Figure 3.

3.2. Adjusting Parameters of $M F R O H$. In order to have the optimal values of $\beta$ and $\Delta$ in the scense of frequency response of MFROH, a proper cost function of ideal low-pass filter must be defined. It is desirable that at low frequencies the amplitude of the frequency response of MFROH is flat and for frequencies higher than $\omega=\omega_{s} / 2$, it is falling sharply. Also it is preferred that the phase of frequency response of $\mathrm{MFROH}$ in passband is flat as much as possible. We assume that the Nyquist sampling condition is satisfied, and therefore passband defines in frequency interval $\left[0,0.1 \omega_{s}\right]$. Optimal $\beta$ and $\Delta$ are those that minimize the following cost function:

$$
\left(\Delta_{\text {opt }}, \beta_{\text {opt }}\right) \triangleq\{(\Delta, \beta): J(\Delta, \beta) \text { is minimum }\},
$$




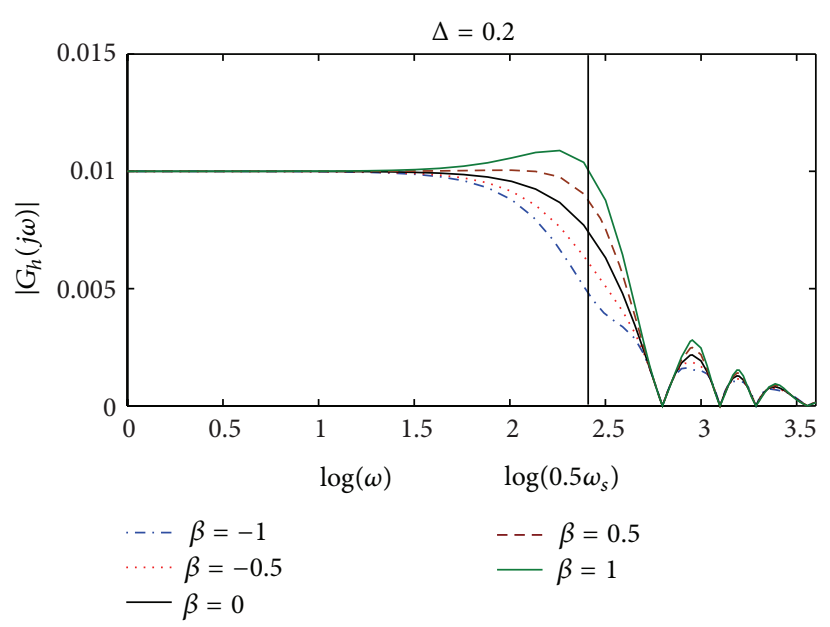

(a)

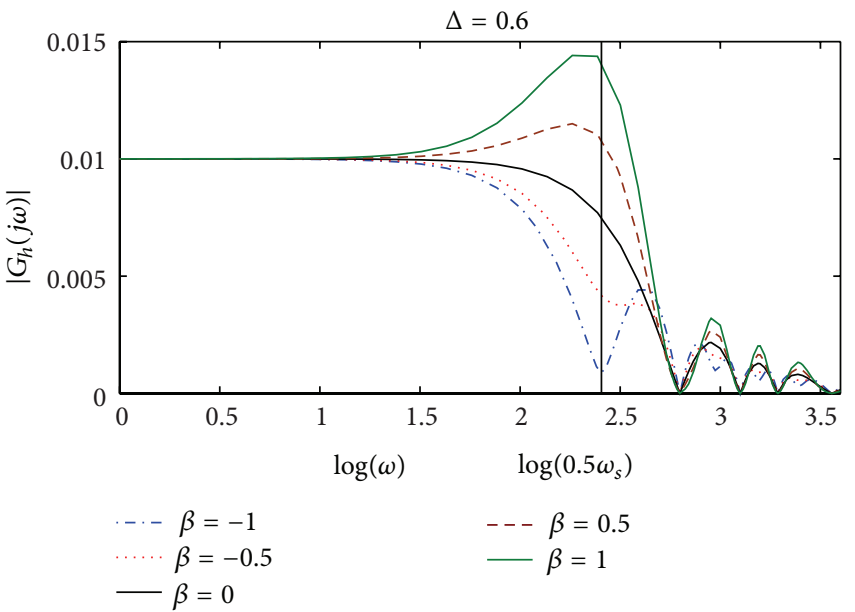

(b)

FIGURE 6: Amplitude of the frequency response of MFROH for different values of $\beta$ and $\Delta$.

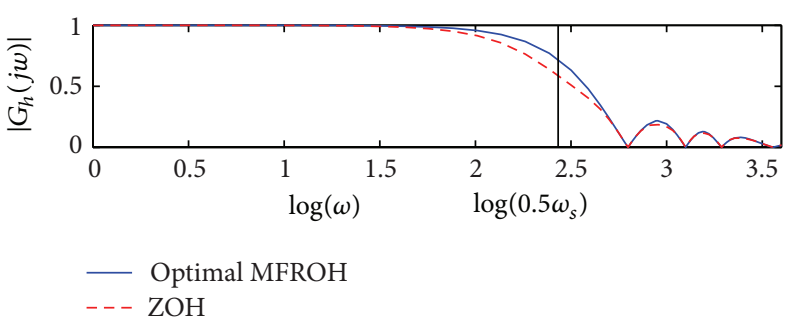

(a)

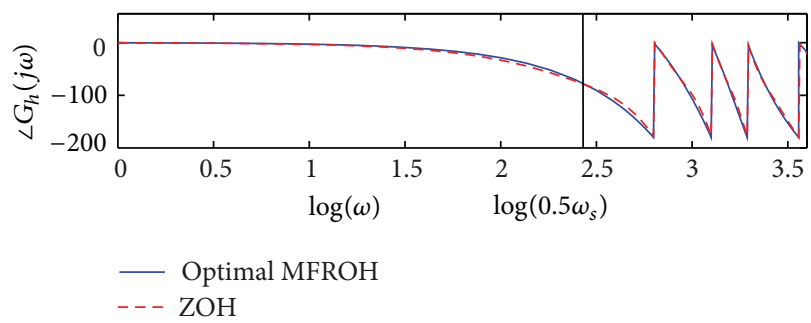

(b)

FIgURE 7: Amplitude and phase of the frequency response of $\mathrm{ZOH}$ and $\mathrm{MFROH}$ with optimal $\beta$ and $\Delta$.

where $J(\Delta, \beta)=\omega_{1} J_{1}(\Delta, \beta)+\omega_{2} J_{2}(\Delta, \beta)+\omega_{3} J_{3}(\Delta, \beta)$. In weighted cost function, $J_{1}(\Delta, \beta), J_{2}(\Delta, \beta)$ and $J_{3}(\Delta, \beta)$ are defined as follows:

$$
\begin{gathered}
J_{1}(\Delta, \beta)=\operatorname{Max}|| G(j \omega)|-| G(j 0) \|, \quad \omega \in\left[0,0.1 \omega_{s}\right], \\
J_{2}(\Delta, \beta)=\operatorname{Max}|\angle G(j \omega)-\angle G(j 0)|, \quad \omega \in\left[0,0.1 \omega_{s}\right], \\
J_{3}(\Delta, \beta)=\operatorname{Max}\|G(j \omega)\|, \quad \omega \in\left[\omega_{s}, \infty\right) .
\end{gathered}
$$

To obtain optimal $\beta$ and $\Delta, \omega_{2}$ is set as $1 / \pi$ and $\omega_{1}$ and $\omega_{3}$ are set to one.

The result of optimization process is obtained as $\beta_{\mathrm{opt}}=$ 0.4 and $\Delta_{\text {opt }}=0.2$ in which the genetic algorithm as a common optimization method is used. The amplitude and phase of the frequency response of MFROH with optimal $\beta$ and $\Delta$ in comparison with frequency response of $\mathrm{ZOH}$ is shown in Figure 7.

As shown in Figure 7, both amplitude and phase of frequency response of optimal MFROH in passband are flatter than those of $\mathrm{ZOH}$.

\section{Stability of Zeros in Sampled System}

About the importance of zeros of sampled system, it is better to consider that the stability properties of the discrete-time zeros do not influence the closed-loop stability, but, as shown in [9], unstable discretized zeros can lead to intersample ripple in some cases. Several techniques for control systems design are based on the cancellation of the system zeros by the controller. Unfortunately, such methods cannot be applied when the system has unstable zeros; see for example [10] and references therein. Therefore the study of the zeros of sampled system is important; it was studied for the $\mathrm{ZOH}$ devices in [11]. Also the stability properties of the FROH zeros (when $T \rightarrow 0$ ) were analyzed by Ishitobi [5].

In this part of the paper, the stability properties of additional generated zeros of MFROH are analyzed. Applying $\mathrm{MFROH}$, it is necessary to adjust parameters of MFROH so that the zeros of the sampled system lie inside the unit circle. However, selecting suitable values for $\beta$ and $\Delta$ for any given sampling period is quite a difficult task. In view of practical control engineering, the sampling period is usually small. Thus, in this paper, we concentrate on the case in which the sampling period $T$ is small. For sufficient small sampling time $T$, the following theorem gives the stability of additional generated zeros of the sampled system in MFROH.

Theorem 1. Suppose that $G_{p}(s)$ is a strictly proper nth-order transfer function and can be expressed as

$$
G_{p}(s)=K \frac{\left(s+b_{1}\right) \cdots\left(s+b_{m}\right)}{\left(s+a_{1}\right) \cdots\left(s+a_{n}\right)}, \quad K \neq 0,
$$


where $a_{i}$ and $b_{i} \in C,(i=1, \ldots, m)$ and $K \neq 0$. Then the $z$ transform of the MFROH combined with the system (5) and for very small $T$ is

$$
\begin{aligned}
H_{\beta, \Delta}(z) & =Z\left\{H_{\beta, \Delta}(s)\right\}=Z\left\{G_{\beta, \Delta}(s) G_{p}(s)\right\} \\
& =K \frac{T^{n-m}}{(n-m) !} \frac{(z-1)^{m} E_{n-m}(z, \Delta, \beta)}{(z-1)^{n} z},
\end{aligned}
$$

where

$$
\begin{aligned}
& E_{n-m}(z, \Delta, \beta) \\
&=(z-\Delta \beta) B_{n-m}(z)+\frac{\beta}{n-m+1} \frac{z B_{n-m+1}(z)}{z-1} \\
& \quad-\frac{\beta}{n-m+1} \frac{B_{n-m+1}(z, \Delta)}{z-1}, \\
& B_{p}(z)= b_{p 1} z^{p-1}+b_{p 2} z^{p-2}+\cdots+b_{p p}, \\
& b_{p k}=\sum_{i=1}^{k}(-1)^{k-i} i^{p}\left(\begin{array}{c}
p+l \\
k-i
\end{array}\right), \quad(k=1, \ldots, p), \\
& B_{p}(z, \Delta)=b_{p 0}(\Delta) z^{p}+b_{p 1}(\Delta) z^{p-1}+\cdots+b_{p p}(\Delta), \\
& b_{p k}(\Delta)=\sum_{l=0}^{p-k}(-1)^{p-k-l}(l+\Delta)^{p}\left(\begin{array}{c}
p+l \\
p-k-l
\end{array}\right), \\
& \quad(k=0,1, \ldots, p),
\end{aligned}
$$

and $\left(\begin{array}{c}n \\ m\end{array}\right)$ indicates $n ! /(n-m) m !$

Note. $B_{n-m+1}(z, \Delta)$ and $B_{n-m+1}(z)$ have term $(z-1)$, and $E_{n-m+1}(z, \Delta)$ is written as (7) for simplicity.

Before proving Theorem 1 , we need some preliminary lemmas.

Lemma 2 (see [4]). The $z$-transform of $\left[\left(1-e^{-s T}\right) / s\right] G_{p}(s)$ for very small $T$ is as follows:

$$
\lim _{T \rightarrow 0} Z\left\{\frac{1-e^{-s T}}{s} G_{p}(s)\right\}=K \frac{T^{n-m}}{(n-m) !} \frac{(z-1)^{m} B_{n-m}(z)}{(z-1)^{n}} .
$$

Lemma 3 (see [4]). The $z$-transform of $\left[\left(1-e^{-s T}\right) / s^{2}\right] G_{p}(s)$ for very small $T$ is as follows:

$$
\begin{aligned}
\lim _{T \rightarrow 0} Z & \left\{\frac{1-e^{-s T}}{s^{2}} G_{p}(s)\right\} \\
& =K \frac{T^{n-m+1}}{(n-m+1) !} \frac{(z-1)^{m} B_{n-m+1}(z)}{(z-1)^{n+1}},
\end{aligned}
$$

where $B_{n-m+1}(z)$ is defined in (8).
Lemma 4 (see [7]). The z-transform of $\left[\left(1-e^{-s T}\right) /\right.$ $\left.s^{2}\right] G_{p}(s) e^{-s \Delta T}$ for very small $T$ is as follows:

$$
\begin{aligned}
\lim _{T \rightarrow 0} Z & \left\{\frac{1-e^{-s T}}{s^{2}} e^{-s \Delta T} G_{p}(s)\right\} \\
= & K \frac{T^{n-m+1}}{(n-m+1) !} \frac{(z-1)^{m} B_{n-m+1}(z, \Delta)}{(z-1)^{n+1} z},
\end{aligned}
$$

where $B_{n-m+1}(z, \Delta)$ is defined in (9).

Proof of Theorem 1. Using the transfer function of MFROH in (2), the transfer function of the discrete system $H_{\beta, \Delta}(s)$ can be expressed as follows:

$$
\begin{aligned}
H_{\beta, \Delta}(s)= & \left\{\frac{\left(1-e^{-s T}\right)\left(1-\Delta \beta e^{-s T}\right)}{s}\right. \\
& \left.+\frac{\beta\left(1-e^{-s T}\right)\left(1-e^{-\Delta s T}\right)}{T s^{2}}\right\} G_{p}(s) \\
= & \left(1-\Delta \beta e^{-s T}\right) \frac{\left(1-e^{-s T}\right)}{s} G_{p}(s) \\
& +\frac{\beta}{T} \frac{\left(1-e^{-s T}\right)}{s} \frac{G_{p}(s)}{s} \\
& -\frac{\beta}{T} \frac{\left(1-e^{-s T}\right)}{s} \frac{G_{p}(s)}{s} e^{-s \Delta T} .
\end{aligned}
$$

The $z$-transform of $H_{\beta, \Delta}(s)$ can be written as follows:

$$
\begin{aligned}
& H_{\beta, \Delta}(z)=Z\left\{H_{\beta, \Delta}(s)\right\}=Z\left\{G_{\beta, \Delta}(s) G_{p}(s)\right\}=\left(1-\Delta \beta z^{-1}\right) \\
& Z\left\{\frac{\left(1-e^{-s T}\right)}{s} G_{p}(s)\right\}+\frac{\beta}{T} Z\left\{\frac{\left(1-e^{-s T}\right)}{s^{2}} G_{p}(s)\right\} \\
&-\frac{\beta}{T} Z\left\{\frac{\left(1-e^{-s T}\right)}{s^{2}} G_{p}(s) e^{-s \Delta T}\right\} .
\end{aligned}
$$

Combining Lemmas 2, 3, and 4 with (14) and after some simplification we would have

$$
H_{\beta, \Delta}(z)=K \frac{T^{n-m}}{(n-m) !} \frac{(z-1)^{m} E_{n-m}(z, \Delta, \beta)}{(z-1)^{n} z} .
$$

Theorem 5. In this theorem one studies the additional generated zeros of the sampled system in $M F R O H$ in which, one assumes that the zeros of the transfer function of the system are minimum-phase in the s-plane.

Case $1(n-m=1)$. The sufficient condition that all zeros of $H_{\beta, \Delta}(z)$ in $0<T \ll 1$ is minimum phase is that all zeros of $G_{p}(s)$ is minimum phase and $\beta$ and $\Delta$ satisfy the following condition:

$$
\beta>\frac{1}{\Delta(\Delta-2)} .
$$


The boundary curve of (16) is as follows:

$$
\beta=\frac{1}{\Delta(\Delta-2)} \text {. }
$$

In $\mathrm{FROH}$ for $n-m=1$, the sufficient condition for stable zeros of $H_{\beta}(z)$ with $0<T \ll 1$ is that all zeros of $G_{p}(s)$ are stable and $-1<\beta[2]$. In this method, the acceptable region of $\beta$ to have stable zeros of sampled system is extended due to the additional parameter $\Delta$. Therefore, with decreasing $\Delta$, the lower bound of $\beta$ increases; that is, for $\Delta=0.1$, zeros of sampled system are stable for $-5.26<\beta$. For $\Delta=1$ as we mentioned before, the behavior of MFROH switches to $\mathrm{FROH}$, and we can see from [5] and (16) that the region of $\beta$ is $-1<\beta$.

Case $2(n-m=2)$. The sufficient condition that all zeros of $H_{\beta, \Delta}(z)$ for $0<T \ll 1$ is minimum phase is that all zeros of $\mathrm{G}_{p}(s)$ is minimum phase and the following conditions are satisfied:

$$
\beta>\frac{1}{\Delta(\Delta-2)}, \quad \beta<0 .
$$

The boundary curve of (18) is as follows:

$$
\beta=\frac{1}{\Delta(\Delta-2)}, \quad \beta=0 .
$$

In FROH for $n-m=2$, the sufficient condition for stable zeros of $H_{\beta}(z)$ with $0<T \ll 1$ is that all zeros of $G_{p}(s)$ are stable and $-1<\beta<0[2]$, whereas in this method, with additional parameter $\Delta$ we can extend the acceptable region of $\beta$ for stability of zeros of sampled system. Note that by decreasing $\Delta$, the range of acceptable $\beta$ increases.

Case $3(n-m \geq 3)$. At least one of the zeros of $H_{\beta, \Delta}(z)$ will be unstable when $0<T \ll 1$, but compared to FROH we can place the non-minimum-phase zeros by adjusting $\Delta$.

Proof of Theorem 5. From Theorem 1, the additional generated zeros of sampled system in $z$-plane can be obtained as follows:

$$
E_{n-m}(z, \Delta, \beta)=0 .
$$

For $n-m=1$, we have

$$
E_{1}(z, \Delta, \beta)=\left(-\frac{1}{2} \beta \Delta^{2}+\beta \Delta+1\right) z+\left(\frac{1}{2} \beta \Delta^{2}-\beta \Delta\right) .
$$

In order to have a stable zero for $E_{1}(z, \Delta, \beta)$, (22) must be satisfied:

$$
\begin{aligned}
\left|\frac{(1 / 2) \beta \Delta^{2}-\beta \Delta}{-(1 / 2) \beta \Delta^{2}+\beta \Delta+1}\right| & <1 \longrightarrow-\beta \Delta^{2}+2 \beta \Delta+1 \\
& >0 \longrightarrow \beta>\frac{1}{\Delta(\Delta-2)} .
\end{aligned}
$$

For $n-m=2$, we have

$$
\begin{aligned}
E_{2}(z, \Delta, \beta)= & \left(1-\beta \Delta^{2}+\beta \Delta+\frac{1}{3} \beta \Delta^{3}\right) z^{2} \\
& +\left(-\frac{2}{3} \beta \Delta^{3}+\beta \Delta^{2}+1\right) z+\left(\frac{1}{3} \beta \Delta^{3}-\beta \Delta\right) .
\end{aligned}
$$

By applying the Jury stability criteria to $(23), E_{2}(z, \Delta, \beta)$, the following constraints must be satisfied:

(1) $E_{2}(1, \Delta, \beta)>0$,

(2) $E_{2}(-1, \Delta, \beta)>0$,

(3) $\left|\left(\frac{1}{3}\right) \beta \Delta^{3}-\beta \Delta\right|<\left|1-\beta \Delta^{2}+\beta \Delta+\left(\frac{1}{3}\right) \beta \Delta^{3}\right|$.

Combination of the above three constraints and after some simplification we have

$$
\begin{gathered}
-\beta \Delta^{2}+2 \beta \Delta+1>0, \\
\beta \Delta^{2}(2 \Delta-3)>0 .
\end{gathered}
$$

In (26), since $0<\Delta<1$, hence, $\Delta^{2}(2 \Delta-3)<0$ will be satisfied for all $\Delta$. Therefore, (25) and (26) will be simplified as (27)

$$
\beta>\frac{1}{\Delta(\Delta-2)}, \quad \beta<0 .
$$

For $n-m=3$ we have

$$
\begin{aligned}
E_{3}(z, \Delta, \beta)= & \left(-\frac{1}{4} \beta \Delta^{4}+\beta \Delta^{3}-\frac{3}{2} \beta \Delta^{2}+\beta \Delta+1\right) z^{3} \\
& +\left(\frac{3}{4} \beta \Delta^{4}-2 \beta \Delta^{3}+3 \beta \Delta+4\right) z^{2} \\
& +\left(-\frac{3}{4} \beta \Delta^{4}+\beta \Delta^{3}+\frac{3}{2} \beta \Delta^{2}-3 \beta \Delta+1\right) z \\
& +\left(\frac{1}{4} \beta \Delta^{4}-\beta \Delta\right) .
\end{aligned}
$$

By applying the Jury stability criteria to $(28), E_{3}(\mathrm{z}, \Delta, \beta)$, the following constraints must be satisfied:

(1) $E_{3}(1, \Delta, \beta)>0$,

(2) $E_{3}(-1, \Delta, \beta)<0$,

(3) $\left|\left(\frac{1}{4}\right) \beta \Delta^{4}-\beta \Delta\right|$

$$
<\left|-\left(\frac{1}{4}\right) \beta \Delta^{4}+\beta \Delta^{3}-\left(\frac{3}{2}\right) \beta \Delta^{2}+\beta \Delta+1\right| .
$$

Since all of the above conditions are not satisfied simultaneously, then $E_{3}(z, \Delta, \beta)$ has at least one unstable zero.

\section{Simulation Results}

In this part, simulation results of the comparison among the proposed holders, $\mathrm{ZOH}$ and $\mathrm{FROH}$, are given. In Figure 8, a continuous system is shown with unity feedback that the sampler and holder is in feedback path.

The simulation result of applying $\mathrm{ZOH}, \mathrm{FROH}$, and MFROM in the holder box in Figure 8 is shown in Figure 9. In Figure 9(a) step response of the closed-loop system is shown, and in Figure 9(b) a special part of the response is 


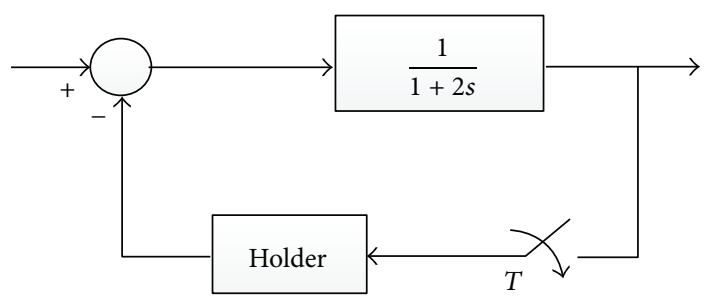

FIgURe 8: A continuous system with a holder.

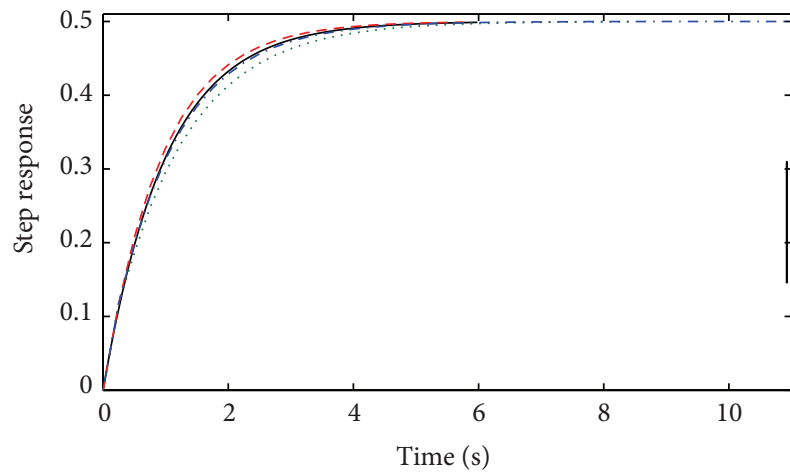

$\begin{array}{ll}\text { - No sampler and holder } \quad \cdots . . \text { FROH } \\ --- \text { ZOH } & \cdots-\text { MFROH }\end{array}$

(a)

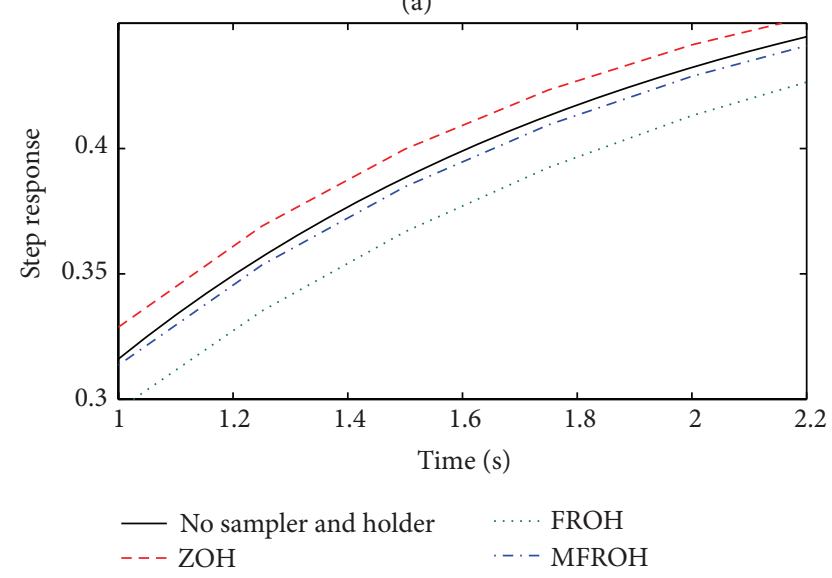

(b)

Figure 9: (a) Step response of the closed-loop continuous system and digital control system when using $\mathrm{MFROH}, \mathrm{FROH}$, and $\mathrm{ZOH}$. (b) Focusing on the step response to show the effect of different holders.

shown to show the difference of the holders. As shown from Figure 9, it is easy to see that the response of the system in MFROM is more similar to the continuous time response (no holder), and it is because of the fact that in MFROH there are two adjusting parameters that using them we can make the response more similar to the continuous time response of the system.

\section{Conclusion}

In this paper we introduced a new holder for digital control systems that has a better performance in terms of the frequency response than $\mathrm{ZOH}, \mathrm{FOH}$, and $\mathrm{FROH}$ and has better frequency response. This new holder has the capability of placing additional generated zeros similar to FROH. But the ranges of adjusting parameters in $\mathrm{MFROH}$ increase, and this is the advantage of MFROH when the holder parameters need to be varied. Simulation results shown in Section 5 show the efficiency of the proposed holder in comparison with typical holders.

\section{References}

[1] C. L. Phillips and H. T. Nagle, Digital Control System Analysis and Design, Prentice Hall, New York, NY, USA, 3rd edition, 1994.

[2] M. Ishitobi, "Properties of zeros of a discrete-time system with fractional order hold," in Proceedings of the 35th IEEE Conference on Decision and Control, pp. 4339-4344, Kobe, Japan, December 1996.

[3] M. J. Blachuta, "On fast sampling zeros of systems with fractional-order hold," in Proceedings of the American Control Conference, Arlington, Va, USA, 1984.

[4] K. J. Åström, P. Hagander, and J. Sternby, "Zeros of sampled systems," Automatica, vol. 20, no. 1, pp. 31-38, 1984.

[5] M. Ishitobi, "Stability of zeros of sampled system with fractional order hold," IEE Proceedings-Control Theory and Applications, vol. 143, no. 3, pp. 296-300, 1996.

[6] M. Ishitobi, "Stability techniques of zeros in discrete-time systems," in Control and Dynamic Systems, C. T. Leondes, Ed., vol. 66, pp. 217-249, Academic Press, New York, NY, USA, 1994.

[7] M. Ishitobi and Q. Zhu, "Zeros of sampled systems with fractional order hold implemented by zero order hold," in Proceedings of the IEEE International Conference on Intelligent Processing Systems (ICIPS '97), vol. 1, pp. 698-702, October 1997.

[8] K. Ogata, Discrete-Time Control Systems, Prentice Hall, New York, NY, USA, 2nd edition, 1994.

[9] M. J. Blachuta, "Continuous-time design of discrete-time control systems," in Proceedings of the European Control Conference, Brussels, Belgium, 1997.

[10] D. W. Clarke, "Self-tuning control of nonminimum-phase systems," Automatica, vol. 20, no. 5, pp. 501-517, 1984.

[11] M. de la Sen, R. Bárcena, and A. J. Garrido, "On the intrinsic limiting zeros as the sampling period tends to zero," IEEE Transactions on Circuits and Systems I, vol. 48, no. 7, pp. 898900, 2001. 


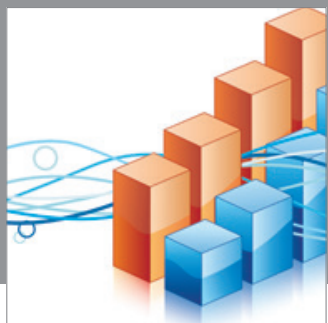

Advances in

Operations Research

mansans

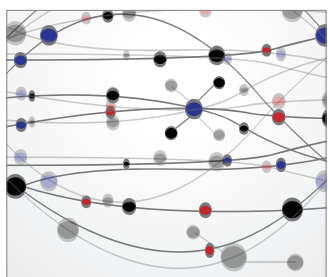

The Scientific World Journal
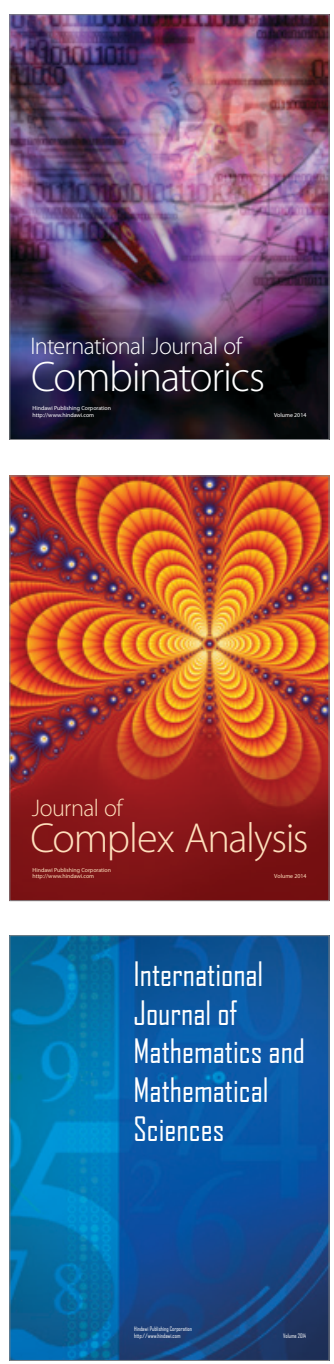
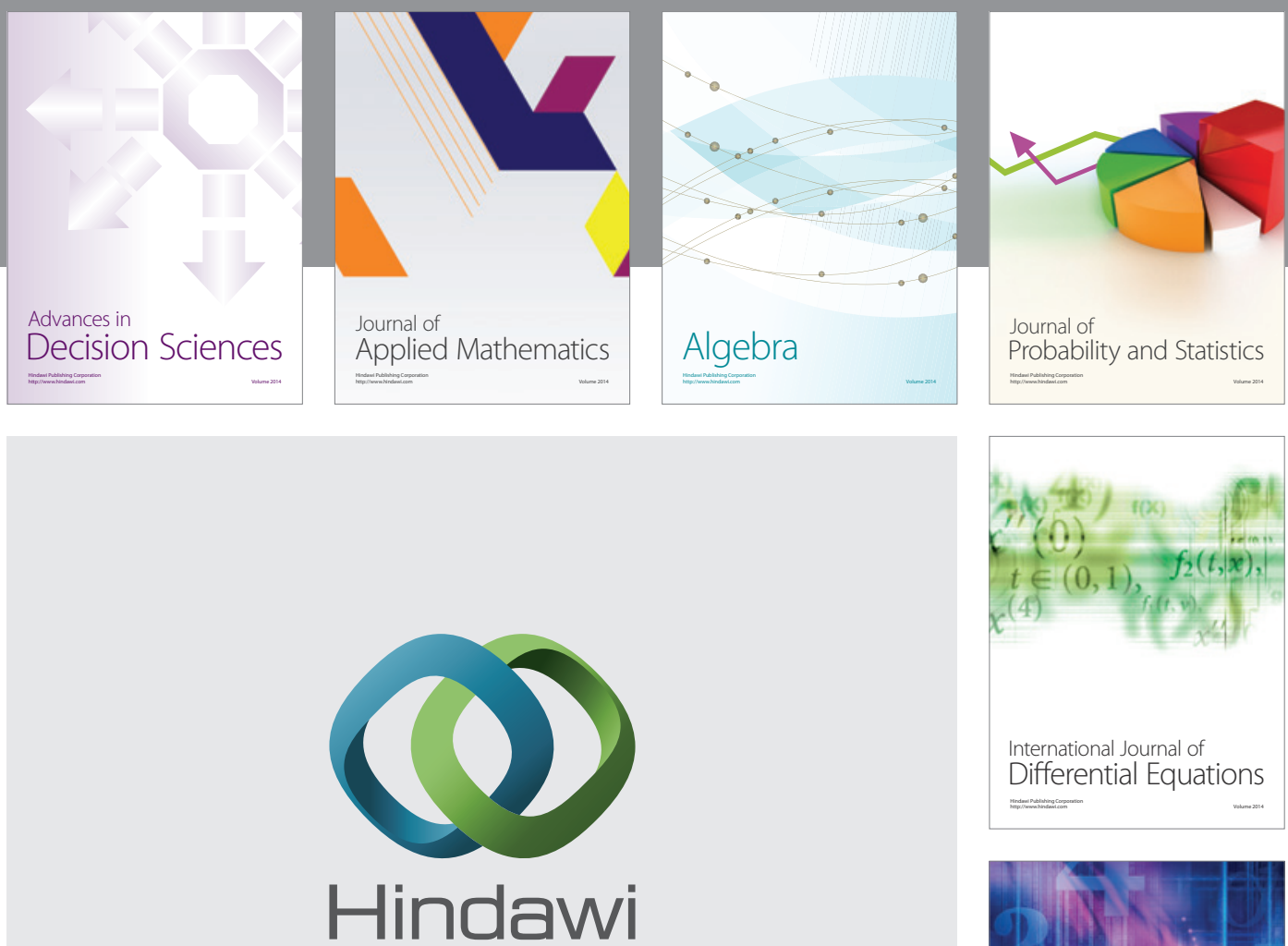

Submit your manuscripts at http://www.hindawi.com
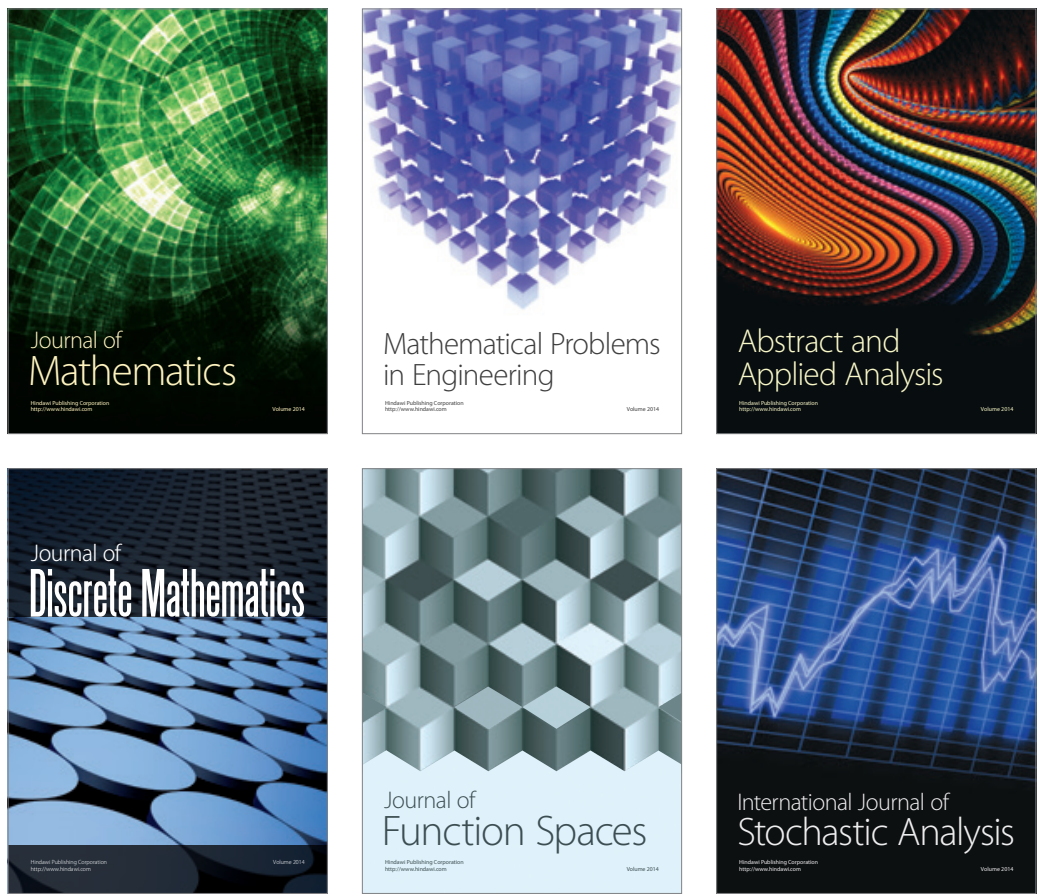

Journal of

Function Spaces

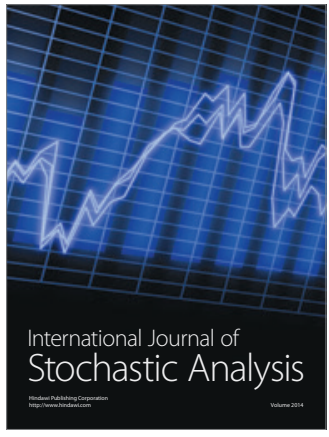

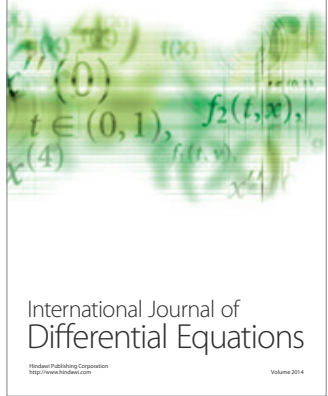
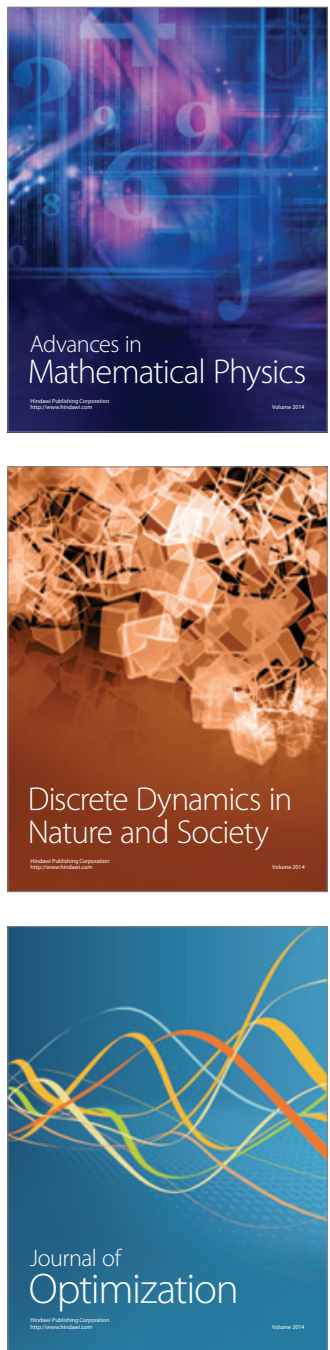